\title{
Privatización del agua y racismo ambiental en ciudades segregadas. La empresa Aguas del Illimani en las ciudades de La Paz y El Alto (1997-2005)/
}

\author{
Water privatization and environmental racism \\ in segregated cities. Aguas del Illimani company \\ in the cities of La Paz and El Alto (1997-2005)
}

Desde el concepto de racismo ambiental aplicado al sector agua, se analiza la concesión del servicio de agua y saneamiento en las ciudades de La Paz y El Alto a la empresa Aguas del Illimani (AISA), filial de la compañía francesa Suez. Ambas ciudades se caracterizan por ser socioeconómica y racialmente segregadas. Se muestra la política de inclusión diferenciada de la empresa respecto a las laderas de la ciudad de La Paz y El Alto, particularmente en los costos de conexión y tarifas del servicio, la exclusión de zonas pobres de aymaras migrantes, los impactos y riesgos ambientales.

PALABRAS ClAVE: Racismo ambiental; Empresa privada de agua; Segregación urbana.

Within the framework of environmental racism in the water sector, the water and sanitation concession contract in the cities of La Paz and El Alto (Bolivia) to Aguas del Illimani company, filial of Suez Group. Both cities are characterised as racially segregated spaces. The differentiated inclusion service policy of the company to neighbourhoods in La Paz and El Alto, particularly costs of connexion and tariffs, the exclusion of poor aymara zones and the environmental risks and impacts.

KEYWORDS: Environmental Racism; Water private Company; Urban Segregation. 


\section{Introducción}

La exclusión indígena y la discriminación social y racial en Bolivia, como dispositivos de dominación económica y política desde la Colonia, han sido señalados desde textos escritos, ${ }^{1}$ así como en el cine y el dibujo. ${ }^{2}$ Esta estructura es considerada como un sistema colonial y de casta; ${ }^{3}$ sus grupos dominantes son definidos como elites patriarcales y coloniales ${ }^{4} \mathrm{o}$ "casta señorial encomendera"; 5 como decía Sergio Almaraz, refiriéndose a la "rosca" minero feudal antes de la Revolución de 1952, estos sectores "viven del país, pero lo desprecian".

El colonialismo interno ha ido moldeando las jerarquías, inclusiones/exclusiones a la vez étnicas y genéricas y ha impedido a estos sectores el acceso a una auténtica ciudadanía. ${ }^{6}$ Más aún, el racismo en Bolivia funciona como un mediador de las relaciones humanas. ${ }^{7}$

La República reprodujo esta estructura de dominación, definida como una república colonial y racista; ${ }^{8}$ como Olivia Harris dijo: "profundas divi-

1 Silvia Rivera Cusicanqui: Oppressed but not Defeated: Peasant Struggles among the Aymara and Qhechwa in Bolivia, 1900-1980, Geneva, UNRISD, 1987; René Zavaleta Mercado: "Las masas en noviembre”. En René Zavaleta (comp.) Bolivia, hoy, México, Siglo XXI Editores, 1983; Soñador Social: "Máquinas sociales autónomas contra el estado racista en Bolivia", ponencia en el II Encuentro del Grupo de Apoyo a los Movimientos Sociales, Quillacollo, 2003.

2 Silvia Rivera Cusicanqui: Invisible Realities: Internal Markets and Subaltern Identities in Contemporary Bolivia, Amsterdam/Quezon City, SEPHIS - SEASREP, 2005. Al respecto ver las películas del cineasta boliviano Jorge Sanjines, Ukamau y Yawar Mallku; o las imágenes de Melchor Pérez Mercado, Album de Paisajes, Tipos Humanos y Costumbres de Bolivia (1841-1869).

3 Silvia Rivera Cusicanqui, "Metáforas"; Carlos Mamani: "Desde el Qullasuyu bajo el signo de la violencia y la muerte", en Bolivian Studies Journal/RevistaE, Vol 4, Issue 1, 2004, pp 7-10, p. 8, http://www.bolivianstudies.org/journal/2004/2004_01.pdf (acceso, 01 de noviembre 2009); Ramón Conde Mamani: "Bolivia colonial y pueblo aymara", en Nicholas A. Robins (ed.): Cambio y continuidad en Bolivia: etnicidad, cultura e identidad, La Paz, Plural Editores, 2005, p. 14. Prada habla de un colonialismo polimorfo, para referir las diversas formas que asume la exclusión indígena (Raúl Prada: "Políticas de las Multitudes", Bolivian Studies Journal/RevistaE, Vol 4, Issue 1, 2004, pp 10-52, p. 45 , http://www.bolivianstudies.org/journal/2004/2004_01.pdf (acceso, 01 de noviembre 2009).

4 Silvia Rivera Cusicanqui: "Metáforas y Retóricas en el Levantamiento de Octubre", Bolivian Studies Journal/RevistaE, Vol 4, Issue 1, February, 2004, pp 3-7, p. 6, http://www.bolivianstudies.org/journal/2004/2004_01.pdf (acceso, 01 de noviembre 2009).

5 Zavaleta, "Las masas ..."

6 Silvia Rivera Cusicanqui: "Los desafíos de una democracia étnica y genérica en los albores del tercer milenio", en S. Rivera (comp.): Ser mujer indígena, chola o birlocha en la Bolivia postcolonial de los años 90, La Paz, Plural Editores/CID, 1996, pp 17-84, p. 19; Rossana Barragán: Indios, mujeres y ciudadanos. Legislación y ejercicio de la ciudadanía en Bolivia (Siglo XIX), La Paz, Fundación Diálogo/Embajada del Reino de Dinamarca en Bolivia, 1999.

7 Guillermo Delgado: "Cincuenta Años Después: El 3 de Agosto de 1953 en una carta informativa", Bolivian Studies Journal/RevistaE, Volumen 3, Issue 2; 2003, pp 50-64, p. 56, http://www.bolivianstudies.org/journal/2003/2003_07.pdf (acceso, 01 noviembre 2009). 
siones sociales heredadas del periodo colonial fueron expresadas en términos de diferencias raciales, y la gente indígena fue vista como un problema". ${ }^{9}$ Por eso la revolución de 1952, si bien ilegalizó la servidumbre y otras formas de explotación casi esclavista e implementó una reforma agraria, ha significado que los indígenas hayan continuado sistemáticamente excluidos de la vida pública y social del país. ${ }^{10}$

Con la modernización neoliberal, las estructuras de dominación se han afianzado y dotado de nuevos mecanismos, cada vez más sutiles, de segregación social y cultural. ${ }^{11}$ Hoy el colonialismo está más vivo que nunca en los Andes, ${ }^{12}$ a pesar de que el neoliberalismo intentó negarlo. Con el Ajuste Estructural, el Estado buscó promover la constitución de una sociedad de actores que consensúan, ${ }^{13}$ incluyente, participativa, basada en el ejercicio pleno de la democracia liberal y el funcionamiento de una economía de mercado. En el marco del modelo neoliberal, según Rivera Cusicanqui, las imágenes de una nación moderna posible y deseable estaban cruzadas por fuerzas divisorias como la etnicidad y la dominación colonial que yace bajo la aparente igualdad provista por el mercado, la ciudadanía y la democracia. ${ }^{14} \mathrm{Al}$ mismo tiempo, las formas cotidianas de discriminación racial perviven en las experiencias de los indígenas de los Andes en áreas urbanas y rurales, definiendo lugares de trabajo, ubicación, pareja, historias y tradiciones. ${ }^{15}$

En Bolivia coexisten y/o se sobreponen la "historia larga" de un estado pre-moderno, autoritario, disciplinario, que ha excluido a la población campesina e indígena, y hoy, también a los pobres urbanos, y una "historia corta" del estado capitalista moderno, liberal, consensualista y biopolítico: Bolivia la "nueva", como decía uno de los slogans del ex-presidente

8 Mamani, "Desde el Qullasuyu...", p. 8.

9 Olivia Harris: To make the earth bear fruit: ethnographic essays on fertility, work and gender in highland Bolivia, London, Institute of Latin American Studies, 2000, p. 3.

10 Robert Albro: "The indigenous in the plural in the Bolivian oppositional politics"; Bulletin of Latin American Research, vol 24, no. 4, 2005, pp. 433-453, p. 434.

11 Rivera Cusicanqui, "Los desafíos de...", p. 18.

12 Conde, "Bolivia colonial...", p. 6.

13 Sobre la crítica a los enfoques consensualistas en el neoliberalismo, ver Carlos Crespo: "La Crisis del Discurso Consensualista de las Políticas Públicas en Bolivia y Conflictos Sociales", en Carlos Vacaflores (ed.): Conflictos y Colaboración en la Gestión de los Recursos Naturales, La Paz, Plural/JAINA/CESU, 2005.

14 Rivera Cusicanqui, Invisible, p. 16.

15 Sara Radcliffe, Nina Laurie y Robert Andolina: "Género y etnodesarrollo en los Andes", en Nina Laurie y María Esther Pozo (eds.): Las Displicencias de Género. Los cruces del siglo pasado al nuevo milenio en Bolivia, Cochabamba, CESU, 2006, pp. 51-96. 
Gonzalo Sánchez de Lozada, principal promotor del modelo neoliberal en el país. El Estado neoliberal boliviano representó la articulación compleja de estas historias, contradictorias y al mismo tiempo complementarias, que coexisten en tensión. ${ }^{16}$

En este marco se analiza la concesión del servicio de agua potable y alcantarillado sanitario, a la empresa Aguas del Illimani (AISA), en las ciudades de La Paz y El Alto, por un contrato de 30 años, firmado en julio de 1997 con el gobierno de Bolivia. AISA era un consorcio internacional dirigido por la compañía francesa Lyonesse des Suez. Luego de la Guerra del Agua de Cochabamba, AISA fue la única concesión privada, hasta su ruptura el año 2006. ${ }^{17}$

El presente trabajo argumenta que en la capital boliviana han operado formas de racismo ambiental, debido a que, por un lado, se ha promovido la profundización de la segregación espacial y exclusión social a los asentamientos humanos pobres, de origen aymara, en el acceso a los servicios de agua y saneamiento, principalmente en la ciudad de El Alto y las laderas de La Paz, a través de un manejo jerárquico de las diferencias y se han generado impactos ambientales sobre el medio ambiente y la calidad de vida de la población, además de incrementar riesgos de desastres naturales, cuyas mayores víctimas han sido los sectores sociales pobres, de origen aymara, visibilizando una forma de racismo ambiental.

En la primera sección se sintetizan los debates sobre el racismo ambiental en el sector agua desde la reducción en el acceso y/o uso de los recursos hídricos y sus servicios, en muchos casos vinculada a procesos de privatización, y los impactos ambientales por actividades relacionadas con el agua. La Paz y El Alto son analizadas como ciudades segregadas racialmente, a la luz de lo cual se muestra la política de inclusión diferenciada de la empresa respecto a las laderas y El Alto, particularmente en los costos de conexión y tarifas del servicio, la exclusión de zonas pobres, los impactos y riesgos ambientales.

Este trabajo es resultado de una beca de la British Academy, y ha sido elaborado en el marco de un programa de postdoctorado en la Universidad de Greenwich en convenio con la Universidad de Newcastle, financiado por la Unión Europea, a través de la Red Alfa "GOVAGUA". En Newcastle,

16 Carlos Crespo: "Respeto tu cultura, pero no te metas con la mía". "Para una crítica del racismo en Bolivia”, Libertaria, n. ${ }^{\circ}$ 1, 2007, pp 37-44.

17 Nina Laurie y Carlos Crespo: "Deconstructing the best case scenario: lessons from La PazEl Alto, Bolivia”, Geoforum, Volume 38, Issue 5, London, 2007, pp 841-854. 
versiones preliminares del documento fueron revisadas por Nina Laurie y José Esteban Castro, quienes dieron recomendaciones valiosas. Asimismo, en Greenwich, los argumentos del documento fueron discutidos con David Hall y Emmanuele Lobinas A todos ellos agradezco el soporte, apoyo y principalmente su amistad.

En mi calidad de miembro del equipo asesor de la FEJUVE El Alto tuve oportunidad de acceder a información valiosa, particularmente las auditorias ambientales y regulatorias; asimismo, participé en reuniones de la organización vecinal de esta ciudad (FEJUVE El Alto) y entre 2004 y 2006, asistí a negociaciones con la empresa y el gobierno, para definir la terminación del contrato. Toda esta etnografía del proceso de terminación del contrato con AISA, ha sido la base del presente documento.

El texto se concentra en el momento de la concesión privada (19972005) y no incluye el proceso de implementación de la empresa pública, en el marco del gobierno del líder indígena Evo Morales como presidente de la república plurinacional de Bolivia, tema que requiere otro análisis.

\section{Racismo ambiental en el sector agua}

Robert Bullard define el racismo ambiental como cualquier política, práctica o directiva, que afecta diferencialmente o crea desventajas (intencionadas o no) a individuos, grupos o comunidades de distinta raza o color. Combina políticas públicas y prácticas de las industrias para proveer beneficios a las corporaciones mientras se trasladan los costos a la población afectada, de origen no blanco. Según Bullard, el gobierno, las instituciones legales, económicas, políticas y militares tienden a reforzar el racismo ambiental e influyen en el uso local del suelo, las regulaciones ambientales, ubicaciones industriales y los lugares donde la gente de color vive, trabaja y juega. ${ }^{18}$ La pregunta de quién paga y quién se beneficia de las políticas industriales y de desarrollo actuales es central en cualquier análisis del racismo ambiental. ${ }^{19}$

18 Robert Bullard: "Environment and morality. Confronting environmental racism in the United States", Programme Identities, Conflict and Cohesion Programme, Paper Number 8, Geneva, United Nations Research Institute for Social Development (UNRISD), 2004, p. 3.

19 Robert. D. Bullard and D. Smith: "Global poverty, pollution, and public health: threats to world security” in Resource Paper (ed.): Second National People of Color Environmental Leadership Summit, Washington D.C., 2003, p. 5. 
Desde la experiencia de países en desarrollo se ha ido más allá del concepto de racismo ambiental aplicado sólo al análisis e intervención de "externalidades" o impactos ambientales producidos por actividades económicas, y se lo ha relacionado también con la privatización y la mercantilización de los recursos naturales y sus servicios, por tanto con una distribución ecológica inequitativa. ${ }^{20}$ De esta manera, propongo hablar de dos tipos de política, práctica o directiva que afectan racialmente a la población, particularmente pobre, en el sector agua y saneamiento:

1.- Reducción en el acceso y/o uso de los recursos hídricos y sus servicios a las poblaciones pobres, de origen indígena, urbanos o rurales, producto de actividades económicas o políticas públicas, incluyendo sobreexplotación de acuíferos en consumo doméstico e industria; $;{ }^{21}$ asimismo, una larga historia de expropiación de la tierra y el agua, como en los EEUU, inspiradas en visiones eurocéntricas, que continúan hasta hoy presentes en los marcos legales e institucionales del agua; ${ }^{22}$ prácticas discriminatorias en la planificación de uso del suelo llevando a severos impactos relacionados con el agua entre comunidades de bajos ingresos y de origen no blanco ${ }^{23}$ prácticas de racismo institucional en la planificación y gestión del agua en países como EEUU ${ }^{24}$ o entre funcionarios del sector público de riego contra comunidades campesinas en países como Perú, ${ }^{25}$ producto del

20 Joan Martínez-Alier: The environmentalism of the poor. A report for the United Nations Research Institute for Social Development (UNRISD) in preparation for the United Nations World Summit on Sustainable Development (WSSD), Johhanessburg, University of Witswatersrand, 2002.

21 W. Braunworth, T. Welch, and R. Hathaway (eds.): Water allocation in the Klamath Reclamation Project, 2001: an assessment of natural resource, economic, social, and institutional issues with a focus on the Upper Klamath Basin, Convallis, Oregon, Oregon State University and the University of California, 2001; Vandana Shiva: Water Wars: Privatization, Pollution and Profit, London, Pluto Press, 2002.

22 Kate Berry: "Race for water? Native Americans, eurocentrism, and western water policy", en David Camacho (ed.): Environmental injustices, political struggles. Race, class and the Environment, Durham \& London, Duke University Press, 1998, pp 125-137.

23 Amy Vanderwarker: "Water, environmental justice and land use planning: Richmond, California”, Progressive Planning, 169, 2006, pp. 26-29 http://www.ejcw.org/PN\%20Fall\%2006\% 20Vanderw.pdf (acceso, 01 de noviembre 2009).

24 Environmental Justice Coalition for Water (EJCW): Environmental justice policy recommendations for CALFED restructure, Oakland, CA, 2005 http://www.lhc.ca.gov/studies/activestudies/ calfed/GuzmanSept05.pdf (acceso, 01 de noviembre del 2009); R. Bath, J. Tansky y R. Villarreal: "The failure to provide basic services to the colonias of El Paso County", David Camacho (ed.): Environmental injustices,..., 1998, pp 101-124.

25 Paul Gelles: Water and power in highland Peru: the cultural politics of irrigation and development, New Brunswick, Rutgers, 2000. 
cual la población no blanca y de bajos ingresos es la más afectada por desastres naturales, como en el caso del huracán Katrina en Nueva Orleans. ${ }^{26}$ La relación entre prácticas racistas hacia minorías indígenas y la falta de acceso adecuado al aprovisionamiento de agua y la introducción de tecnologías de agua, ha sido destacada. ${ }^{27}$ En países con estructuras sociales basadas en castas, como la India, poblaciones ubicadas en la base de la pirámide social son excluidas del acceso a fuentes del recurso agua potable y saneamiento del agua. ${ }^{28}$ La inequidad en el acceso al agua, basada en el origen étnico entre la población palestina y la árabe de Israel, ha sido también analizada. ${ }^{29}$

En EEUU existen activistas ambientales que relacionan racismo y acceso al agua, como el Environmental Justice Coalition for Water, ${ }^{30}$ que trabaja empoderando miembros de comunidades para convertirse en voces defensoras de la justicia del agua en sus comunidades, particularmente en población no blanca y de bajos ingresos.

La privatización de los servicios de agua y saneamiento incluye cambios organizativos, en la propiedad (de lo público a lo privado) y en el manejo (del casi total control público de las funciones de manejo del agua al involucramiento del sector privado). ${ }^{31}$ Con el fondo de la privatización del agua, se observan dos tipos de políticas, prácticas o directivas, en el sector, que afectan a la población más pobre, de manera vinculada a la raza:

- Los impactos ambientales y riesgos generados por actividades económicas, afectando a poblaciones pobres, quienes asumen los costos y efectos ambientales en su vida diaria, por ejemplo, la contaminación

26 Environmental Justice Coalition for Water.

27 Federal Race Discrimination Commissioner: Water: a report on the provision of water and sanitation in remote aboriginal and Torres Strait islander communities, Canberra, AGPS, 1994.

28 S, Narula and M. Macwan: "Untouchability': the economic exclusion of the Dalits in India"; paper presented to the Seminar on the Economics of Racism, The International Council on Human Rights Policy, Geneva, January 2001, 10 pp. http://www.ichrp.org/files/papers/158/113__Untouchability_-_The_Economic_Exclusion_of_the_Dalits_in_India_Narula_Smita_Macwan__ Martin_2001.pdf (acceso, 1 de noviembre 2009).

29 José E. Castro: Transboundary water sharing and conflict: review of best practice and its application to the Middle East and North Africa Region: the socio-political dimension, reporte inédito, Newcastle upon Tyne, Newcastle University, 2007a.

30 http://www.ejcw.org/index.html, acceso 01 de noviembre 2009.

31 Karen Bakker: "From state to market?: water mercantilización in Spain", Environment and Planning A, 34(5), London, 2002, pp 767-790, p. 768. 
hídrica producida por la industria, ${ }^{32}$ los hidrocarburos, ${ }^{33}$ minería, ${ }^{34}$ afectando la salud de la población pobre no blanca ${ }^{35}$ asimismo, las expresiones del movimiento de justicia ambiental y ecologismo de los pobres $^{36}$ contra la construcción de grandes presas debido a sus impactos contra la población y comunidades locales. ${ }^{37}$

- La reducción en el acceso y uso de los recursos hídricos y sus servicios, producto de actividades económicas o políticas públicas. Shiva ha analizado el caso de la empresa Coca Cola, y los impactos negativos de sus operaciones en países en desarrollo, en su mayoría comunidades locales/indígenas pobres. ${ }^{38}$

Martínez Alier compara las luchas de los movimientos urbanos por el agua en Sudáfrica contra la mercantilización y privatización del agua y saneamiento, con los levantamientos de Bolivia y Argentina. ${ }^{39}$

2.-Impactos y riesgos ambientales generados por actividades relacionadas con el agua, las cuales son descargadas, directa o indirectamente, sobre poblaciones indígenas o no blancas, quienes asumen en su vida cotidiana sus efectos y costos ambientales, por ejemplo la contaminación hídrica producida por la industria, ${ }^{40}$ industria química ${ }^{41}$ o minería ${ }^{42}$ afectando la salud de gente pobre no blanca ${ }^{43}$ Existen varios trabajos acerca del movimiento de justicia ambiental y el ecologismo de los pobres ${ }^{44}$ contra la cons-

32 J. N. Clarke y A. K. Gerlak: "Environmental racism in the Sunbelt: a cross-cultural analysis", Environmental Management 22. 6, 1998, pp. 857-867.

33 I. Salinas: Observing and absorbing: a case of environmental racism. A brief historical analysis on the adverse impacts of corporate agribusiness and chemical production in Mission, Texas, Mission, Texas, Mission Texas Coalition/Mexican American Studies, 2006.

34 Thabo Madihlaba: "The fox in the henhouse: the environmental impact of mining on communities", en David. A. McDonald (ed.): Environmental justice in South Africa, Athens \& Cape Town, Ohio University Press/University of Cape Town Press, 2002, pp. 156-168.

35 Bullard: "Environment and morality...".

36 Martínez-Alier, The environmentalism of the poor...

37 Patrick Bond: "A political economy of dam building and household water supply in Lesotho and South Africa", en David A. McDonald (ed.): Environmental Justice in South Africa, 2002, pp 223269; Patrick McCully: Silenced rivers: the ecology and politics of large dams, London, Zed Books and International Rivers Network, 1996.

38 Shiva, Water Wars...

39 Joan Martínez-Alier: Ecological distribution conflicts in a context of uncertainty, Tenerife, FRONTIERS-ESEE/University of La Laguna, 2003, p. 12.

40 Clarke y Gerlak, "Environmental racism ...".

41 Salinas, Observing and absorbing...

42 Madihlaba, "The fox in the henhouse...".

43 Bullard, "Environment and morality..."

44 Martínez-Alier, The environmentalism of... 
trucción de grandes presas debido a sus impactos sociales y ambientales sobre poblaciones y comunidades locales. ${ }^{45}$

Uno de los rasgos del sector agua y saneamiento es la tendencia a internalizar los beneficios económicos y socializar los costos sociales y ambientales, producto de sus actividades; el sector privado privatiza los beneficios obtenidos y los distribuye entre sus socios o propietarios, pero no asume las externalidades o impactos ambientales generados por estas actividades, y los transfiere a la sociedad, quien debe asumirlos. Pero, en países en desarrollo con fuerte presencia indígena, en muchos casos constituyendo verdadera mayoría, como en Bolivia, no es toda la sociedad quien asume los costos ambientales, sino los sectores más pobres, principalmente de origen indígena, quienes sufren los mayores efectos de tales impactos, expresión de una "distribución ecológica inequitativa"; 46 este es uno de los rasgos más importantes de la privatización del agua en países como Bolivia.

\section{Segregación espacial, privatización del agua y racismo ambiental en $\mathrm{La} \mathrm{Paz} / \mathrm{El}$ Alto}

Las ciudades de La Paz y El Alto forman parte de la mayor área metropolitana de Bolivia (La Paz-El Alto-Viacha-Achocalla) con 839,594 y $832,312^{47}$ habitantes en 2005 , respectivamente. ${ }^{48}$ La Paz esta situada en un valle estrecho, entre los 2.800 y 4000 metros sobre el nivel del mar, rodeada de montañas. El centro de la ciudad se extiende de norte a sur, y está rodeado por laderas de hasta $50 \%$, en el este y oeste, densamente pobladas, ${ }^{49}$ en su mayoría por migrantes aymaras. Mientras, la ciudad de El Alto se encuentra en una amplia altiplanicie contigua a La Paz.

45 Bond, "A political economy...”; McCully, Silenced rivers...

46 Martínez-Alier, The environmentalism of...

47 Para el 2006, se calcula que hay alrededor de 800.000 habitantes sólo en El Alto (Raúl Zibechi: El Alto: A World of Difference, IRC Americas, 2006, p. 1 http://americas.irc-online.org/ pdf/reports/0510elalto.pdf (acceso, 01 de noviembre de 2009).

48 http://www.ine.gov.bo/indice/visualizador.aspx?ah=PC20402.HTM (acceso, 02 de noviembre de 2009)

49 Fabien Nathan: Risk perception, risk management and vulnerability to landslides in the hillslopes in the city of La Paz, Bolivia. A preliminary statement. Paper presented to UNU-EHS Summer Academy in Munich, 23-30 July, 2006, p. 3. 
La migración se ha traducido en segregación, no sólo socioeconómica, como hemos visto, sino también espacial. ${ }^{50} \mathrm{El}$ Alto no es únicamente el enclave más pobre de la región metropolitana de La Paz, sino un espacio racializado, distante de la ciudad moderna y más sofisticada de La Paz, ${ }^{51}$ particularmente la zona sur; El Alto "es una ciudad de indios" como comentó un ciudadano paceño a un investigador. ${ }^{52}$

Siguiendo la definición de David Theo Goldberg, ${ }^{53}$ podríamos decir que la ciudad de El Alto y las laderas de La Paz constituyen espacios racialmente segregados, pues mientras las elites políticas y económicas - también denominados "jailones" - viven en la parte central y sur de la ciudad de La Paz, predominantemente blanca, los sectores pobres, conformados básicamente por migrantes de origen aymara, viven en las laderas y El Alto. ${ }^{54}$ Los aymaras que viven en los vecindarios de ambas ciudades se hallan en peores condiciones de vida frente a los "jailones", 55 de hecho, la segregación espacial está negativa y significativamente referida al ingreso; más aún, ser aymara y vivir en un vecindario segregado está clara y negativamente correlacionado con la educación. ${ }^{56}$ Las laderas de La Paz funcionan como barrera física entre la zona central y la ciudad de El Alto. AISA operaba en estos dos espacios urbanos segregados racialmente

\section{Costos, segregación y racialización del servicio de agua y saneamiento}

En la presente sección se analizan dos aspectos de los efectos de la privatización del agua en el acceso y uso al agua y sus servicios, particularmente en poblaciones pobres, de origen no blanco, el enfoque punitivo

50 Ibidem, p. 5.

51 Juan Arbona: Ver y hacer política en la ciudad de El Alto. Capacidades políticas y actividades económicas, La Paz, PNUD, 2002, pp. 4-5.

52 "Los alteños son vistos como personas peligrosas, como delincuentes" (Ibidem, p. 5).

53 David Theo Goldberg: Racist Culture. Philosophy and the politics of meaning, Oxford \& Cambridge, Blackwell, 1994, p. 193.

54 "One could say that on the Northern Plateau, the poor live above (El Alto) and the rich live below (La Paz)" (Zibechi, El Alto..., p. 1).

55 George Gray Molina, E. Pérez de Rada y W. Jiménez: Social Exclusion: Residential Segregation in Bolivian Cities; Washington, Fundación Diálogo/Inter-American Development Bank Research Network working papers ; R-440; 2002, 31 pp., p. 17.

56 Ibidem, p. 19. 
de las tarifas, la exclusión o inclusión diferenciada del servicio, reproduciendo procesos de segregación espacial, y finalmente los efectos en los derechos tradicionales de agua.

\section{Costos de conexión y tarifas: técnicas para excluir a poblaciones pobres no blancas}

AISA consideraba que una vivienda tenía el "servicio" si la tubería central estaba instalada en la calle, sin importar si existían acometidas domiciliarias; de esta manera, al menos 68.400 personas en El Alto no lograron conectarse, a pesar de que vivían en zonas donde la red de agua potable había sido implementada, y no lo habían hecho debido fundamentalmente al incremento de costos de conexión, en más de $26 \%$ en agua y $38 \%$ en alcantarillado, al inicio del segundo quinquenio de la concesión (2001-2006), ${ }^{57}$ afectando a las familias pobres de esa ciudad.

En algunos países en desarrollo, las tarifas están indexadas a tasas de cambio internacional; en La Paz/El Alto, y antes en la concesión de Cochabamba al consorcio Aguas del Tunari, ${ }^{58}$ lo estaban al dólar americano, incluyendo incrementos automáticos de acuerdo a los cambios de la paridad de esa moneda con la boliviana y el nivel de inflación en los EEUU. En Bolivia, los salarios no están indexados al dólar americano, por tanto, un incremento en las tarifas a través de la indexación, representaba una pérdida en la capacidad adquisitiva de la población pobre de estas ciudades.

\section{Segregación espacial en la gestión privatizada del agua}

Otro aspecto del racismo ambiental está relacionado con las prácticas de inclusión diferenciada en la expansión del servicio. El sector privado usualmente no está interesado en proveer el servicio de aquellas áreas más pobres, por no ser económicamente rentables. ${ }^{59}$ Para ello, se introducen cláusulas en los contratos destinadas a excluir aquellas áreas. ${ }^{60}$ En la con-

57 Laurie \& Crespo, "Deconstructing the best case ...".

58 Carlos Crespo: Water Policies and Conflicts: The Water War in Cochabamba (1999/2000), tesis para doctorado, Oxford, Oxford Brookes University, 2003.

59 Ibidem.

60 Laurie \& Crespo, "Deconstructing the best case ...". 
cesión de El Alto/La Paz, se introdujo una peculiar distinción entre área de concesión y área servida, la primera referida a la concesionada por la autoridad regulatoria boliviana, que incluía todo el territorio de los municipios de El Alto y La Paz, mientras que el área servida sólo abarcaba los límites geográficos donde SAMAPA (la empresa municipal de agua previa a la concesión) trabajó originalmente. El tamaño del área servida era menor que el área concesionada. AISA rechazó dar atención a la población fuera del área servida, excepto si los vecinos cubrían el costo total o era un proyecto financiado externamente. Bajo esta cláusula del contrato, vecindarios pobres periurbanos de El Alto, migrantes aymaras, sólo podían tener acceso al servicio pagando el costo total. En una ciudad con un rápido crecimiento demográfico como El Alto, en muchos casos sin aprobación de las mismas autoridades locales, significó excluir al 16\% de la población alteña, esto es casi 130.000 personas. ${ }^{61}$

\section{Privatización del agua y la racialización de los impactos y riesgos ambientales}

AISA no sólo vulneró aspectos del contrato y las normas vigentes sino que generó impactos ambientales, muchos de ellos graves, cargó los costos ambientales a la población, particularmente la más pobre de origen aymara, e incrementó los riesgos de desastres naturales y sobre la calidad del entorno ambiental y la población. ${ }^{62}$ En ese sentido, la FEJUVE El Alto denunciaba que "la falta de mantenimiento y la no reposición oportuna de tuberías, válvulas y otros componentes que están desgastados, además de la construcción de Sistemas Condominiales baratos con el objetivo de ahorrar dinero a Aguas del Illimani, acaban provocando focos de contaminación en viviendas y zonas de El Alto". ${ }^{63}$ Veamos las principales expresiones.

Plantas de Tratamiento.-A lo largo de la concesión, tres de las cuatro plantas de Tratamiento de agua potable (Achachicala, Pampahasi y El Alto) descargaron diversas sustancias por encima de lo establecido en el Reglamento en Materia de Contaminación Hídrica (RMCH), de la Ley de

\footnotetext{
61 Ibidem.

62 Pozo \& Asociados C.P.A S.R.L, Auditoría Regulatoria ...”, $2006^{\mathrm{a}}$.

63 FEJUVE El Alto (2004): 14 Razones para romper el contrato con Aguas del Illimani, La Paz, FEJUVE, 2004, p. 14.
} 
Medio Ambiente, ${ }^{64}$ desde sólidos suspendidos, zinc (en algunos casos excedieron en $4233 \%$ ), hierro (con exceso entre 1100 y $1100 \%$ ), hasta cromo, en algún momento; mientras que los valores DQO se excedieron hasta en $354 \%$. Todas ellas se encuentran en zonas deprimidas y segregadas de ambas ciudades.

Descarga de lodos.-La planta de tratamiento de Achachicala descarga anualmente al río Choqueyapu $184.737 \mathrm{~m} 3$ de lodos, conteniendo metales pesados; ${ }^{65}$ entre 1998 y 2005, se calcula que se descargaron 5 toneladas de cianuro, 45 de plomo, 41 de arsénico, 5 de cadmio, 95 de cobre, 14.172 de hierro, 323 de manganeso y 1.539 de zinc $^{66}$. Estas aguas posteriormente son utilizadas, entre otros, en actividades agropecuarias, por agricultores aymaras, río abajo. La causa del alto contenido de metales pesados es que una de las fuentes utilizadas (Milluni) es un área minera abandonada, por tanto con pasivos ambientales. AISA, en el plan de inversiones propuesto al inicio de la concesión, debía cambiar de fuente, justamente por los riesgos ambientales y para la salud que supone consumir aguas de relaves mineros, pero no lo hizo.

Planta de Tratamiento de Aguas Servidas Puchuckollo.-Esta planta se halla ubicada en el municipio de Laja, cerca de la ciudad de El Alto, aunque con un índice de desarrollo humano de 0.563 (cuando el municipio de la ciudad de La Paz tiene 0.730), ${ }^{67}$ uno de los más pobres de la ciudad de El Alto. No sólo recibe aguas servidas de origen doméstico, sino también aguas residuales industriales y lixiviados.

Existe una fuente de contaminación atmosférica, a través de la emisión de sulfuro de hidrógeno (H2S). Entre 2003 y 2005, en la planta de Puchuckollo, la concentración de $\mathrm{H} 2 \mathrm{~S}$ en la planta sobrepasó el nivel de límites permisibles establecido en el reglamento ambiental, entre $4 \mathrm{y}$ $23.3 \%$, y esta "se viene incrementando", afectando al "factor atmosférico local". ${ }^{68}$ Un plan de reforestación implementado por AISA para reducir los impactos fracasó, por falta de mantenimiento.

Asimismo, AISA, en diversos momentos e intensidades, realizó descargas por encima de la norma, conteniendo coliformes fecales (con exce-

\footnotetext{
64 Pozo y Asociados, C.P.A S.R.L, Auditoría Regulatoria...2006a , pp.14-28.

65 Ibidem, p. 51.

66 Ibidem, p. 55.

67 Programa de Naciones Unidas para el Desarrollo -PNUD: Indice de desarrollo humano de los municipios de Bolivia, La Paz, PNUD, 2004.

68 Pozo \& Asociados C.P.A S.R.L: Auditoría Regulatoria con carácter Técnico Ambiental,... La Paz, 2006a, pp. 31, 33.
} 
sos entre $3500 \%$ y $11900 \%$ ), amonio (el exceso oscilaba entre $305 \%$ y $2441 \%$ ); DBO con 10 a $65 \%$ por encima de la norma y DQO con $29 \%$ por encima de la norma, en el 2005. Del 2000 al 2005 la planta descargó 43 toneladas de fósforo y 1578 Ton de nitrógeno. ${ }^{69}$ Las descargas llegaron hasta el lago Titicaca, a través del río Pallina, el cual a su vez tiene como afluentes al Río Seco (en cuyo recorrido recibe el efluente contaminado de Puchuckollo), Hernani y Seke, también contaminados. Los mayores impactos fueron sobre comunidades campesinas aymaras pobres:

- La contaminación contribuyó al proceso de eutrofización de la Bahía de Aygachi del Lago, ${ }^{70}$ amenazando a patos y animales silvestres andinos como la ch'oc'a, k'ellwa, tiki, wisch'ocho, unk'alla, sulu, q'ueñu" ${ }^{71}$ y afectando a por lo menos 17 comunidades aymaras, ${ }^{72}$ que viven de la pesca, ${ }^{73}$ agricultura y ganadería, dando a esos animales forraje con plantas de totora contaminadas. ${ }^{74}$ Asimismo, el turismo de la zona, fuente importante de ingresos para la región, fue amenazado.

- En su curso, la contaminación afectó "el uso de estas aguas para la actividad de pastoreo, riego y cultivo, entre otras". ${ }^{75}$

- Consumo humano. En la ruta al lago, comunidades muy pobres como Pallina, que sobreviven sin servicios básicos, alcantarillado y menos agua potable, utilizan las aguas contaminadas del río para sus actividades cotidianas. ${ }^{76}$

Los impactos generados por la planta de Puchuckollo se deben a que, por un lado, ésta ha sido sobrepasada en su capacidad, y por otro, a que al no existir un sistema pluvial, el agua de lluvia es descargada directamente en el alcantarillado ${ }^{77}$.

69 Ibidem, p. 98.

70 Ibidem, p. 35.

71 www.bolpress.com; 07 de julio 2006.

72 Ibidem, 21 de marzo, 2006.

73 Las pesquerías del lago Poopó, que en el pasado llegaban a rendimientos de hasta 3.000 toneladas métricas por año, hoy han visto reducida su producción, debido a la contaminación acuática, particularmente el ingreso y consiguiente aumento de niveles de sal y metales pesados en el lago www.bolpress.com, 07 de Julio 2006.

74 www.bolpress.com; 31 de mayo 2006.

75 Pozo \& Asociados C.P.A S.R.L, Auditoría Regulatoria ..., $2006^{\mathrm{a}}$, p. 32.

76 www.bolpress.com; 21 de marzo 2006.

77 Contraloría General de la República: Informe de Auditoria ambiental No KL/AP43/M02. sobre los sistemas de abastecimiento de aguas potable y alcantarillado de las ciudades de La Paz, El Alto y Alrededores. La Paz, 2002, pp. 31-32. 
Pasivos ambientales._-Los efectos de los impactos ambientales sobre las poblaciones pobres se agravan si tomamos en cuenta que los impactos arriba señalados, en algunos casos provenían de periodos previos a la concesión, sobre la cual la empresa no realizó ninguna acción, como la ya mencionada fuente de agua de Milluni, pues de acuerdo al contrato de concesión, AISA no se hacía cargo de los pasivos ambientales existentes a la fecha de inicio (18.1.5.). ¿Quién asume estos costos ambientales, pasados y futuros? Los pobladores afectados. ${ }^{78}$ Un tema adicional son los impactos acumulativos pues, como se sabe, a pesar de que los indicadores puedan estar por debajo de los estándares permitidos, en el largo plazo pueden acumularse, en el cuerpo humano o receptor.

Red Pluvial.-AISA logró que en el contrato no se incluyera la red pluvial como responsabilidad, excepto el mantenimiento de la red existente. ${ }^{79}$ Para la ciudad de La Paz, cuyas laderas, mayoritariamente pobladas por inmigrantes aymaras pobres, se hallan en alto riesgo de derrumbes durante la época de lluvias, la inexistencia de una política de manejo pluvial es grave. Para AISA el tema pluvial no generaba ganancias, por tanto no constituía una prioridad. Pero esta empresa ni siquiera cumplió con la responsabilidad de mantener el sistema pluvial existente. ${ }^{80}$ El hecho se agrava si tomamos en cuenta que "el sistema de drenaje pluvial del centro de la ciudad (de La Paz) ha superado la vida útil”, infraestructura que debía ser renovada por AISA, como parte de las actividades de mantenimiento.

El 19 de febrero del 2002, como resultado de una violenta inundación $^{81}$ en el centro de la ciudad de La Paz, hubo un saldo de 77 personas fallecidas, 103 heridos - la mayoría de ellos pequeños comerciantes y vendedores callejeros, de origen aymara, que fueron sorprendidos por la riada- y más de diez millones de dólares en pérdidas y daños. ${ }^{82}$ Semanas antes, a propósito de una inundación previa en esta parte de la ciudad, el alcalde de La Paz, Juan del Granado, denunció que la causa fue que AISA no limpió los sumideros. ${ }^{83}$

78 La preocupación por los pasivos ambientales está reflejada en una carta de Guillermo Arroyo, ex -gerente de la empresa, a la autoridad regulatoria, el 5 de abril de 2005.

79 Laurie \& Crespo, "Deconstructing the best case ...".

80 Morrison y Asociados: Propuesta para el mejoramiento del sistema pluvial en el centro de la ciudad de La Paz, La Paz, Mimeo, 2004.

81 Ese día, se calcula que hubo una intensidad de precipitación de $41 \mathrm{~mm} / \mathrm{hora}$ (desde las 14:20 a las 15:45) (Morrison y Asociados, Propuesta...).

82 Ibidem.

83 El Diario, 17 de enero de 2002. 
Pérdidas de agua.-Entre el 2003 y el 2004, las zonas más pobres y de mayor riesgo de derrumbes, El Alto y las laderas, también tuvieron las mayores pérdidas en las redes de distribución (entre un 34 y un $38 \%$ en El Alto y 47 al $50 \%$ en las laderas); mientras que en los sistemas de Achachicala y Pampahasi, donde se hallan el centro de la ciudad y los barrios de elites, las pérdidas fueron menores (entre el 14 y el $28 \%$ y entre el 22 y el $26 \%$, respectivamente). ${ }^{84}$

\section{Conclusiones}

1.-En el sector agua, distingo dos tipos de racismo ambiental: uno, los impactos y riesgos ambientales generados por actividades económicas, costos que son cargados o asumidos, directa o indirectamente, sobre poblaciones indígenas o no blancas pobres.

2.- - La reducción en el acceso y uso de recursos naturales (hídricos en el caso que nos convoca), producto de actividades económicas o de políticas públicas.

Las políticas neoliberales en el sector agua, implementadas en países en desarrollo, promovieron la privatización de los servicios de agua y saneamiento. En países con mayoría de población indígena o no blanca, este proceso incluyó ambos tipos de racismo ambiental.

La empresa AISA operó practicando y/o reproduciendo formas de racismo ambiental; para dar evidencia de ello, se han estudiado dos aspectos relacionados con la concesión, como el espacio y el medio ambiente:

Espacio._La Paz y El Alto son ciudades racialmente segregadas, con una población pobre mayoritariamente aymara, viviendo en las laderas de la primera y en El Alto; muchas de estas zonas no fueron consideradas por la filial de Suez parte de sus responsabilidades, basándose en una distinción ente área de concesión y área servida, establecida en el contrato. AISA sólo operó en el área servida y, aunque no se los excluyó totalmente, los consideró como una zona especial, como criterio de manejo, que implicó excluir a casi el $16 \%$ de la población alteña en el acceso al servicio (casi 130.000 personas). De la misma manera, interpretando una cláusula del contrato, la

84 Pozo y Asociados: Auditoría Regulatoria con carácter Técnico - Ambiental, Económico Financiera, Legal y Comercial sobre el cumplimiento del Contrato de Concesión de Aguas del Illimani S.A.. Otros temas priorizados. Recursos hídricos, La Paz, 2006b, pp. 8-9. 
empresa consideró como vivienda servida aquella donde la tubería central estaba instalada en la calle, sin importar si los vecinos estaban o no conectados. De esta manera, al menos 68.400 personas fueron excluidas del servicio, a pesar de vivir dentro la llamada área "servida". En total 200.000 personas, dentro y fuera del área servida, fueron excluidas; esto es casi el $25 \%$ de la población de El Alto, cifra que crece en unas 30.000 personas al año.

Ambiente.-Los impactos y riesgos ambientales producidos por la concesión, en muchos casos incumpliendo aspectos del contrato, visibilizan el caso como racismo ambiental. Los graves impactos de las plantas de tratamiento, tanto de aguas potables como residuales, las descargas de lodos y la existencia de pasivos ambientales, incluyendo la descarga de metales pesados, que son depositados en ecosistemas conservados como el lago Titicaca y afectan el hábitat de comunidades aymaras, urbanas y rurales, han sido evidenciados por la auditoría regulatoria realizada por el gobierno boliviano en el 2006. Por otro lado, AISA no cumplió con su responsabilidad de mantener la red pluvial existente, ${ }^{85}$ incrementando los riesgos de desastres naturales en una ciudad como La Paz, con graves problemas de derrumbes en las zonas pobres de las laderas, por la infiltración del agua en época de lluvias y la inexistencia de un apropiado sistema de descarga pluvial, evidenciado en una fatal inundación ocurrida en el 2002 en la ciudad de La Paz, matando a 77 personas, causando 103 heridos y daños por más de 10 millones de dólares. AISA no sólo produjo impactos ambientales, vulnerando normas y regulaciones ambientales, sino que los costos y riesgos sociales y ambientales fueron cargados a los sectores más pobres de El Alto y las laderas de La Paz, como parte de una política de manejo jerárquico de las diferencias en relación a la zona central y sur de la ciudad de La Paz. Al sector privado, como AISA, le interesa fundamentalmente privatizar beneficios y socializar los costos ambientales a la sociedad, siendo los pobres los más afectados.

3.-Las condiciones para facilitar las prácticas de racismo ambiental señaladas, estaban en muchos casos legitimadas por el contrato de concesión, como la distinción entre área servida y área de concesión, por ejemplo. La autoridad regulatoria aceptó esas condiciones favorables a la empresa, y ésta reprodujo esta lógica racializada de gestión pública. Así, el caso AISA reproduce la mentalidad de los poderes dominantes en Bolivia,

85 Morrison y Asociados, Propuesta ... 
para quienes el indígena, urbano o rural, ha sido considerado un sujeto inferior, biológica y culturalmente, en una escala jerárquica racializada y de dominación. El Estado se ha constituido desde esta visión. El proyecto modernizador del neoliberalismo reprodujo este esquema y al mismo tiempo lo adecuó a los tiempos, a través del discurso multiculturalista, como formas culturalistas del racismo. ${ }^{86}$

4.-En la historia ecológica de Bolivia, los pobres, principalmente indígenas, dentro y fuera de las ciudades, han sido los que han sufrido los efectos del desarrollo y la modernización sobre su hábitat, a través de la destrucción de recursos y espacios de vida, y la contaminación de sus territorios, planteándose un grave caso de justicia ambiental. El neoliberalismo reprodujo esta tendencia de la historia larga, y el contrato de concesión lo visibilizaba en los alcances, en la calidad del servicio; lo grave es que, como se ha mostrado, la filial de Suez ni siquiera cumplió aspectos ambientales del contrato, afectando así a las laderas y El Alto.

Por ello el desafío para el proceso iniciado por el gobierno del líder indígena Evo Morales, él mismo de origen aymara, de modificar el racismo ambiental de la ahora empresa pública del agua y saneamiento de La Paz y El Alto (EPSAS), es inmenso, y sobre él se abre un campo de investigación importante. 